\title{
Reconfigurable nanophotonic cavities with nonvolatile response
}

Jan von Keitz ${ }^{1}$, Johannes Feldmann ${ }^{1}$, Nico Gruhler ${ }^{1}$, Carlos Ríos ${ }^{2,3}$, C. David Wright ${ }^{4}$, Harish Bhaskaran $^{2}$, Wolfram Pernice ${ }^{1}$

${ }^{1}$ Institute of Physics and Center for Nanotechnology, University of Münster, 48149 Münster, Germany

${ }^{2}$ Department of Materials, University of Oxford, Parks Road, Oxford, OX1 3PH, UK

${ }^{3}$ Department of Materials Science \& Engineering, Massachusetts Institute of Technology, Cambridge, MA 02139, USA

${ }^{4}$ Department of Engineering, University of Exeter, Exeter, EX4 QF, UK

\section{ABSTRACT:}

The use of phase-change materials on waveguide photonics is presently being purported for a range of applications from on-chip photonic data storage to new computing paradigms. Photonic integrated circuits in combination with phase-change materials provide on-chip control handles, featuring non-volatility and operation speeds down to the nano- and picosecond regime. Besides ultrafast control, efficient operation of non-volatile elements is crucial and requires compact photonic designs. Here we embed phase-change materials in photonic crystal cavities to realize tunable nanophotonic devices which can be reconfigured on demand. The devices exploit strong light matter interactions between the resonant modes of the cavity and the evanescently coupled phase-change material cell. This results in a increased transmission contrast and a power reduction of $520 \%$ over conventional phasechange nanophotonic devices when reversibly switched with optical pulses. Such designs can thus open up new areas of reconfigurable nanophotonics without sacrificing the speeds or functionality for applications in optical memory cells, optical switches and tunable wavelength filters. 


\section{KEYWORDS: photonic crystal cavity, phase-change materials, integrated optics}

Modern communication and computation systems are moving towards fully optical operation because high-speed transmission rates provide an attractive solution to overcoming bandwidth limitations of electronic signaling. Whereas long-range communication is comprehensively accomplished in the optical domain, time and energy consuming electro-optical conversions are necessary for subsequent data processing with electronic integrated circuits. Silicon photonics which offers solutions to realize optical interconnects between individual components [1], [2] is not only paving the way towards substituting electro-optical conversions with all-optical processing, but also shows promise to overcome the limited data interchange rate between the system memory and the central processing unit (CPU) of modern computer systems (von-Neumann bottleneck) [3], [4]. Promising integrated photonic data processing elements such as low power switches [5] or random access memories [6] have recently been developed in this context. However, these devices are volatile and require a steady optical bias, continuously consuming energy to preserve their state. In contrast, on-chip photonic structures in combination with phasechange materials (PCMs) enable non-volatile operation without biasing. PCMs, whose discovery in 1968 is attributed to Stanford Ovshinsky [7], feature significantly distinct optical and electronic properties whether the material is dominantly in the amorphous or crystalline phase of matter. Not only fast switching times in the sub-nanosecond regime between phase states [8] and the ability to remain stable at room temperature for decades guaranteeing full non-volatility, but also high cyclability [9] are outstanding features of PCMs.

When integrated with photonic waveguides, phase-change materials can be used as an active component to modulate the transmitted light, because the imaginary part of the refractive index changes (in the near infrared) by more than an order of magnitude upon switching. In waveguide devices, nanoscale PCM cells are closely coupled to propagating optical modes and interact 
through the evanescent near-field. Phase transitions can be triggered via short optical pulses, which induce appropriate heat stimuli due to absorption of light [10]. Corresponding to the phase state ratio of crystalline and amorphous parts of the PCM, distinct effective absorption coefficients are generated. Consequently, a wide tunability of transmission levels can be exploited for non-volatile and all-optical applications such as multi-level memory [11], all-optical switches [12]-[14] or even all-optical calculations [15]. Overall, a low energy consumption for operation and a high contrast of distinct transmission levels are favorable features, which can be improved significantly by integrating the PCM cell into resonant structures [14], [16].

Here, we demonstrate an integrated, all-optical and non-volatile reconfigurable wavelength selective element based on a photonic crystal cavity in combination with the PCM $\mathrm{Ge}_{2} \mathrm{Sb}_{2} \mathrm{Te}_{5}$ (GST) evanescently coupled to the center of the cavity (Figure 1). By actively controlling the phase state of the GST element with optical nanosecond pulses (red), the low transmission profile of a dominantly crystalline GST cell (orange) can be transformed into a high transmission cavity of a dominantly amorphous phase state (blue) and vice versa. The difference in transmission is on the one hand caused by a high absorption coefficient of crystalline GST, while on the other hand the wavelength shift of the resonance mode due to refractive index change of the PCM element further increases the contrast, as illustrated in the transmission profiles (Figure 1). The fabricated device provides a tunable change in transmission with a very high contrast of up to $14 \mathrm{~dB}$ and shows high cyclability.

The lifetime of a resonance mode within a photonic crystal cavity is related to its Q-factor, given by the ratio of the stored energy and the energy dissipated per resonating cycle. Scattering out of the cavity and strong localization of the mode should be reduced to a minimum to achieve high-Q resonant modes. In order to design a cavity with the aim to minimize scattering loss in the axial 
direction we keep the period $a$, i.e. the distance between holes, constant to ensure optical phase matching between adjacent unit cells [17]. The out-scattering loss perpendicular to the surface of the cavity is minimized if the Fourier components of the electric and magnetic field are located at the edge of the Brillouin zone, i.e. furthest away from the light line [18]. By generating a Gaussian shaped field profile within the cavity, the Fourier components are effectively pushed towards the edge of the Brillouin zone [19]. A convenient way to generate a Gaussian field profile is to linearly increase the attenuation of the electromagnetic field from the center to the outside of the cavity. To achieve this the fill factor, which is the ratio of the hole radius and surface of each unit cell, is tapered quadratically along the cavity as described by Quan et al. [20].

The design goal is a high-Q photonic crystal cavity in order to boost the interaction between guided modes in the telecom regime and the evanescently coupled GST cell. Hence, the hole period is mainly optimized to guarantee operation in the C-band, while the fill factor and number of holes are important parameters to minimize scattering losses. It will later be shown that a deposited GST cell alters the resonance condition of the cavity due to distinct refractive indices of the different phase states of the GST. It induces a shift of the resonance wavelength leading to a tunable contrast in transmission. In Figure 2 (a), simulated field profiles obtained from finite difference time domain (FDTD) simulations of the first, second and third order resonance modes along a cavity with $N=$ 50 holes per side and a period $a$ of $520 \mathrm{~nm}$ are presented. The FDTD simulations reveal that the first order resonance mode exhibits the desired Gaussian shaped profile with maximal field strength at the center of the cavity, where the GST cell will be placed later on. Similarly, tt can be seen that modes with the order $\mathrm{n}$ feature $\mathrm{n}-1$ nodes, resulting in a minimum in field strength at the center of the cavity for even modes. Therefore, less optical damping due to the presence of the GST is expected for cavity modes with odd symmetry. 
The transmission spectrum in Figure 2 (b) for a fabricated device without GST in the cavity center with similar parameters compared to the simulation (Figure 2 (a)) shows that transmission for wavelengths lower than $1562 \mathrm{~nm}$ is close to zero due to the band gap of the cavity.Within the bandgap, light is mainly back-reflected. Light on resonance is transmitted and corresponds to peaks or dips in the transmission or reflection spectrum. Since the peak power on resonance in the transmitted spectrum almost reaches the level of the reflected power off resonance the losses introduced by the cavity itself are low. The main loss channels leading to reduced transmission on resonance are scattering losses introduced by fabrication imperfections and surface roughness of the waveguide. The resonance peaks at $\lambda_{\text {res., },}=1562 \mathrm{~nm}, \lambda_{\text {res., } 2}=1571 \mathrm{~nm}$ and $\lambda_{\text {res., },}=1581 \mathrm{~nm}$, correspond to the simulated mode profiles with $\lambda_{\text {res., } \mathrm{S} 1}=1563 \mathrm{~nm}, \lambda_{\text {res., }} \mathrm{S} 2=1572 \mathrm{~nm}$ and $\lambda_{\text {res., }} \mathrm{s} 3=$ $1581 \mathrm{~nm}$, representing the first, second and third order resonance mode with Q-factors of 7500, 1400 and 440, respectively. In Figure 2 (c) it is shown experimentally that the Q-factor can be enhanced exponentially by increasing the number of holes to improve confinement of the mode in axial direction. Q-factors higher than 100000 for $N=90$ have been obtained before deposition of GST. Fabricated devices show the ability to modify the resonant wavelength of a cavity by changing the period $a$ in Figure 2 (d). Each resonance wavelength increases linearly with increasing period. Consequently, a period between $510 \mathrm{~nm}$ and $520 \mathrm{~nm}$ allows operation within the telecom C-band.

The SEM micrograph in Fig 3 (a) exemplifies a functional fabricated photonic crystal cavity, including the sputter-deposited GST cell marked in green. The period is in this case $520 \mathrm{~nm}$ and the number of holes per side 75 . The PCM cell with a film thickness of $10 \mathrm{~nm}$ has a length of $l_{\mathrm{GST}}$ $=0.91 \mu \mathrm{m}$ covering the whole waveguide width of $1.2 \mu \mathrm{m}$. A layer of $10 \mathrm{~nm} \mathrm{SiO} 2$ is deposited on top of the PCM to prevent oxidation. The device is subsequently analyzed in different phase states. 
The corresponding transmission spectrum presented in Figure 3 (b) compares the cases of fully amorphous (after sputter deposition) and fully crystalline GST. It has been fully crystallized by using a hotplate to heat up the sample above the glass-transition temperature for more than $10 \mathrm{~min}$. Overall, the transmission values for the amorphous case are higher, because the absorption coefficient is lower in this case. In the crystalline state, in particular the transmission of the third order resonance mode is influenced more strongly compared to the second or fourth order modes due to the phase-transition. By recalling the mode profiles (Figure 2 (a)) it becomes clear that odd modes experience stronger optical absorption effects, since they exhibit a maximum field in the center of the cavity where the PCM is located. Hence, the interaction with the cavity modes can be tailored by the depositing the GST cell at suitable locations of the cavity. Because of the interaction with the GST, the resonance wavelengths of the cavity depend on phase transitions and corresponding changes of the refractive index. While odd modes show a positive shift when switching the GST from the amorphous to the crystalline state $\left(\Delta \lambda_{\text {res., }} 3=0.32 \mathrm{~nm}\right)$, even modes shift to lower wavelengths $\left(\Delta \lambda_{\text {res., } 2}=-0.18 \mathrm{~nm}, \Delta \lambda_{\text {res., } 4}=-0.17 \mathrm{~nm}\right)$. We note that, differing from the transmission spectrum of the device without GST (Figure 2 (b)), the first order resonance mode is not present in the measured response. This is due to the fact that light on resonance is fully absorbed due to the large interaction with the PCM. By inspecting the second order resonance mode in more detail in Figure 3 (c), it can be seen that the wavelength shift plays a significant role for the contrast in transmission between the crystalline and amorphous phase of the GST. The wavelength dependent contrast values for the comparison of a fully crystalline cell with a fully amorphous (as deposited) cell show a maximum of $14.3 \mathrm{~dB}$. However, it is challenging to reach the fully crystalline and fully amorphous states in a dynamic experiment when switching with optical pulses and therefore this value has to be viewed as an upper boundary for the switching contrast. 
To change the phase state of the PCM with light pulses sent down the photonic crystal waveguide, the switching scheme utilizing pulses with a width of $100 \mathrm{~ns}$ is presented in Figure 4 (a). A single pulse with $100 \%$ intensity, which is high enough to partly melt the GST cell, followed by fast heat dissipation and the resulting quenching process, is used to amorphize parts of the PCM [21]. To recrystallize the cell a train of five consecutive pulses (each pulse repeated five times) with decreasing intensity is used. Due to the lower absorption coefficient of now amorphous cell domains, absorption is reduced. Hence, the PCM is heated above the glass-transition temperature while staying below the melting temperature. The increased mobility of atoms above the glasstransition temperature allows reverting into the energetically more favorable crystalline phase [10]. By using a pulse train with decreasing pulse energies, reamorphization of PCM cell is prevented. The operation wavelength used to switch the PCM in the cavity is the resonance wavelength (1555 $\mathrm{nm}$ for this device). Due to the moderate $\mathrm{Q}$ factor with embedded GST, the resonances in the amorphous and crystalline state still overlap and thus enable switching in both directions using the same wavelength. The readout of the state is performed at a wavelength of $1542 \mathrm{~nm}$. We note that a similar scheme can be created using pulses in the picosecond regime with the aim to further minimize the switching time and energy [12].

In Figure 4 (b) the correlation between energy consumption to switch the cell into the dominantly amorphous phase and maximal switching contrast is presented for a GST cell embedded on a conventional photonic waveguide $\left(l_{\mathrm{GST}, \mathrm{wg}}=1.04 \mu \mathrm{m}\right)$ and for a GST cell embedded in the center of a photonic crystal cavity $\left(a=510 \mathrm{~nm}, l_{\mathrm{GST}, \mathrm{pc}}=0.91 \mu \mathrm{m}\right)$. The switching contrast is defined as the change in transmission between the mostly crystalline and mostly amorphous phase state of the cell acquired by the use of the presented switching scheme. It is apparent that high switching contrast of a GST cell embedded in the cavity requires significantly lower power values compared 
to a cell on a conventional waveguide. In addition, the switching contrast for the cell in the cavity greatly exceeds the values for a cell on a waveguide. The conventional waveguide design consumes $360 \mathrm{pJ}$ to achieve a contrast of $0.1 \mathrm{~dB}$, while for the cavity design, energy reduction of $14 \%$ (310 pJ) with a concurrent increase in contrast of $3.3 \mathrm{~dB}$ is apparent. To achieve a similar contrast value of around $1 \mathrm{~dB}$, the cavity design consumes only $19 \%$ of the energy consumed by the waveguide design. The graph in Figure 4 (b) further illustrates that the switching contrast benefits from the wavelength shift, because with increasing pulse energy the shift increases in the same manner as the contrast. During these experiments the highest wavelength shift was measured to be $0.13 \mathrm{~nm}$ for the pulse energy of $310 \mathrm{pJ}$.

The enhanced contrast and decrease in energy consumption is achieved with the photonic crystal cavity, because of a more efficient absorption process due to recirculation of light within the cavity. The absorption process starts uniformly from the middle of the cavity, where the field strength is maximal (Figure 2(a)). For GST on top of a conventional waveguide, a passing pulse only affects the cell once and the absorption process starts at the beginning of the cell, which leaves rear cell parts largely unaffected. Additionally, the reconfiguration of the cavity improves the contrast values (Figure 3 (c) and 4 (b)). Because of the variation of the refractive index of the GST the resonance condition of the cavity changes, leading to a shift of the resonance wavelength. Finally, we show in Figure 4 (c) the high cyclability of our PCM-based approach, in this case demonstrating that the switching contrast is constant for multiple (here more than 2200) switches to the high transmission state and back again to the low transmission state. However, high cyclability up to $10^{12}$ switches has already been shown for phase-change materials highlighting the excellent reversibility of the phase transition [9]. 
In this work we have shown that not only the contrast values upon switching can be increased by integrating a GST cell into a photonic crystal cavity instead of operating with a cell on a bare waveguide, but also that the energy consumption (for switching) can be reduced drastically. To achieve a contrast of $1 \mathrm{~dB}$ the consumption is diminished by a factor higher than 5 (Figure 4 (b)) for similar GST cells. Therefore, our device leads the way for more effective and more efficient non-volatile all-optical control handles in the area of photonic integrated circuits. Promising improvements can be envisaged for example in the field of optical memory cells, tunable wavelength filters since the contrast is strongly wavelength dependent and optical switches or tunable mirrors. 


\section{ASSOCIATED CONTENT}

\section{Supporting Information}

Measurement setup

Simulations -Parameters for the cavity design

Fabrication

Energy consumption

\section{ORCID}

\section{Notes}

The authors declare no competing financial interest. 


\section{References}

[1] M. Paniccia, "Integrating silicon photonics," Nat. Photonics, vol. 4, no. 8, pp. 498-499, 2010.

[2] C. Sun, M. T. Wade, Y. Lee, J. S. Orcutt, L. Alloatti, M. S. Georgas, A. S. Waterman, J. M. Shainline, R. R. Avizienis, S. Lin, B. R. Moss, R. Kumar, F. Pavanello, A. H. Atabaki, H. M. Cook, A. J. Ou, J. C. Leu, Y.-H. Chen, K. Asanović, R. J. Ram, M. A. Popović, and V. M. Stojanović, "Single-chip microprocessor that communicates directly using light," Nature, vol. 528, no. 7583, pp. 534-538, 2015.

[3] A. Alduino and M. Paniccia, "Interconnects: Wiring electronics with light," Nat. Photonics, vol. 1, no. 3, pp. 153-155, 2007.

[4] R. Kirchain and L. Kimerling, "A roadmap for nanophotonics," Nat. Photonics, vol. 1, no. 6, pp. 303-305, 2007.

[5] E. Kuramochi, K. Nozaki, A. Shinya, K. Takeda, T. Sato, S. Matsuo, H. Taniyama, H. Sumikura, and M. Notomi, "Large-scale integration of wavelength-addressable all-optical memories on a photonic crystal chip," Nat. Photonics, vol. 8, no. 6, pp. 474-481, 2014.

[6] K. Nozaki, A. Shinya, S. Matsuo, Y. Suzaki, T. Segawa, T. Sato, Y. Kawaguchi, R. Takahashi, and M. Notomi, "Ultralow-power all-optical RAM based on nanocavities," Nat. Photonics, vol. 6, no. 4, pp. 248-252, 2012.

[7] S. R. Ovshinsky, "Reversible electrical switching phenomena in disordered structures," Phys. Rev. Lett., vol. 21, no. 20, pp. 1450-1453, 1968.

[8] D. Loke, T. H. Lee, W. J. Wang, L. P. Shi, R. Zhao, Y. C. Yeo, T. C. Chong, and S. R. Elliott, "Breaking the speed limits of phase-change memory," Science, vol. 336, no. 6088, pp. 1566-1569, 2012.

[9] G. W. Burr, "Phase change memory technology," J. Vac. Sci. , vol. 2004, no. 2, p. 903, 2010.

[10] M. Wuttig and N. Yamada, "Phase-change materials for rewriteable data storage," Nat. Mater., vol. 6, no. 11, pp. 824-832, 2007.

[11] C. Rios, M. Stegmaier, P. Hosseini, D. Wang, T. Scherer, C. D. Wright, H. Bhaskaran, and W. H. P. Pernice, "Integrated all-photonic non-volatile multi-level memory," Nat. Photonics, vol. 9, no. 11, pp. 725-732, 2015.

[12] M. Stegmaier, C. Ríos, H. Bhaskaran, C. D. Wright, and W. H. P. Pernice, "Nonvolatile all-optical $1 \times 2$ switch for chipscale photonic networks," Adv. Opt. Mater., vol. 5, p. 
$1600346,2017$.

[13] D. Tanaka, Y. Shoji, M. Kuwahara, X. Wang, K. Kintaka, H. Kawashima, T. Toyosaki, Y. Ikuma, and H. Tsuda, "Ultra-small, self-holding, optical gate switch using Ge 2 Sb 2 Te 5 with a multi-mode Si waveguide," Opt. Express, vol. 20, no. 9, pp. 10283-10294, 2012.

[14] M. Rudé, J. Pello, R. E. Simpson, J. Osmond, G. Roelkens, J. J. G. M. Van Der Tol, and V. Pruneri, "Optical switching at $1.55 \mu \mathrm{m}$ in silicon racetrack resonators using phase change materials," Appl. Phys. Lett., vol. 103, no. 14, p. 141119, 2013.

[15] J. Feldmann, M. Stegmaier, N. Gruhler, C. Ríos, H. Bhaskaran, C. D. Wright, and W. H. P. Pernice, "Calculating with light using a chip-scale all-optical abacus," Nat. Commun., vol. $8,2017$.

[16] J. I. Z. Heng, A. M. E. Y. K. Hanolkar, P. X. U. Eipeng, S. H. C. Olburn, S. A. D. Eshmukh, J. A. M. Yers, J. E. F. Rantz, E. R. I. C. P. Op, J. O. H. Endrickson, J. O. D. Oylend, and N. I. B. Oechler, "GST-on-silicon hybrid nanophotonic integrated circuits : a non-volatile quasi- continuously reprogrammable platform," vol. 8, no. 6, pp. 1551-1561, 2018.

[17] Q. Quan, P. B. Deotare, and M. Loncar, "Photonic crystal nanobeam cavity strongly coupled to the feeding waveguide," Appl. Phys. Lett., vol. 96, no. 20, pp. 94-97, 2010.

[18] K. Srinivasan and O. Painter, "Momentum space design of high-Q photonic crystal optical cavities," Opt. Express, vol. 10, no. 15, p. 670, 2002.

[19] Y. Akahane, T. Asano, B. Song, and S. Noda, "High- Q photonic nanocavity in a twodimensional photonic crystal," Nature, vol. 425, no. October, p. 944, 2003.

[20] Q. Quan and M. Loncar, "High-Q, low index-contrast polymeric photonic crystal nanobeam cavities," Opt. Express, vol. 19, no. 19, pp. 18529-18542, 2011.

[21] M. Stegmaier, C. Rios, H. Bhaskaran, and W. H. P. Pernice, "Thermo-optical Effect in Phase-Change Nanophotonics," ACS Photonics, vol. 3, no. 5, pp. 828-835, 2016. 


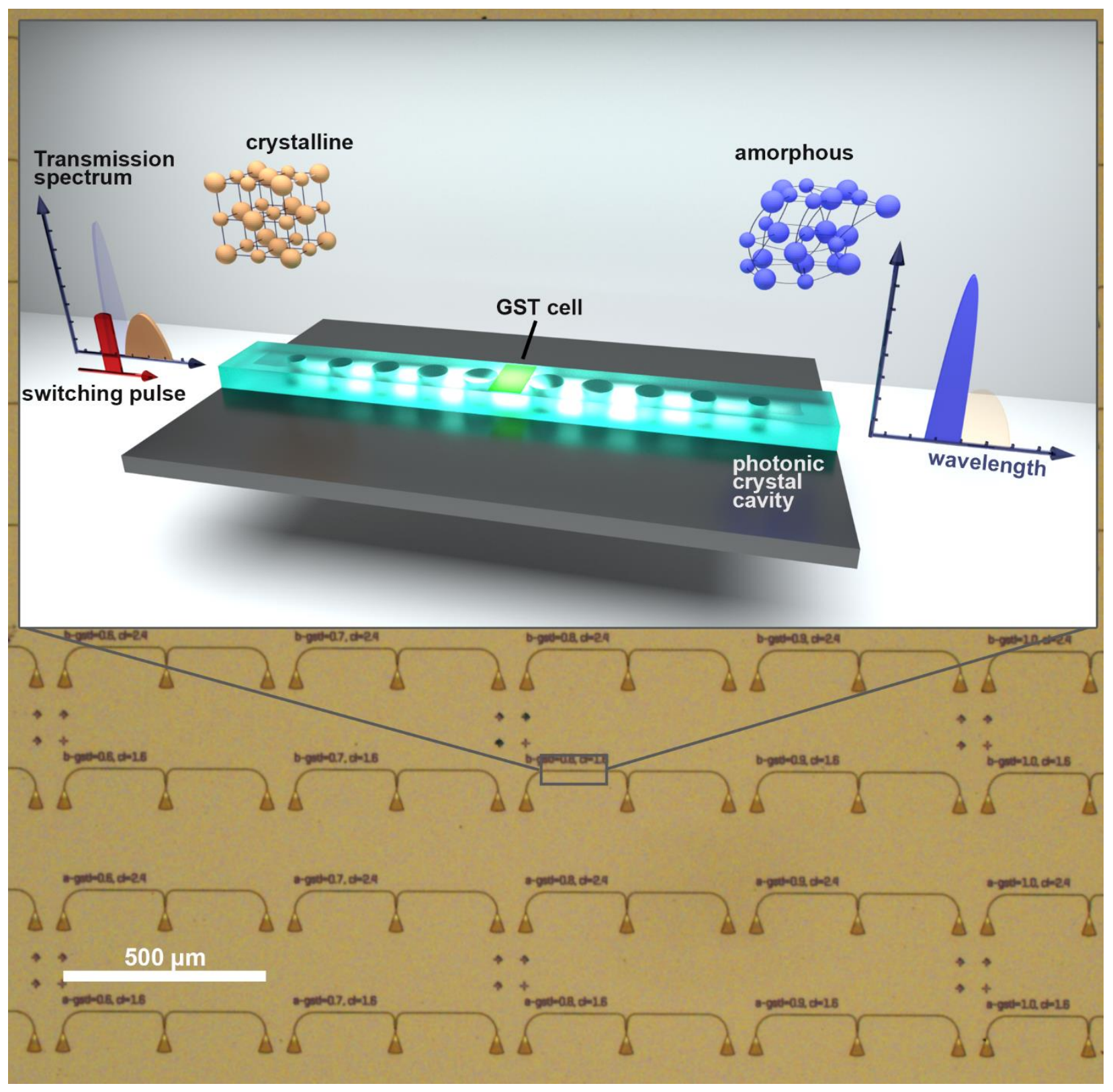

Figure 1. Optical micrograph of on-chip photonic circuits used in this work. The inset shows an illustration of a photonic crystal cavity that can non-volatilely and all-optically be reconfigured due to the GST cell, which is evanescently coupled to the center of the cavity. For a crystalline GST cell, the transmission is low. The resonance wavelength and transmission can be modified by amorphizing the cell with the help of optical switching pulses. 
Second order resonance mode $\left(\lambda_{\text {res }, 2}\right)$

Third order resonance mode $\left(\lambda_{\text {res, } 3}\right)$
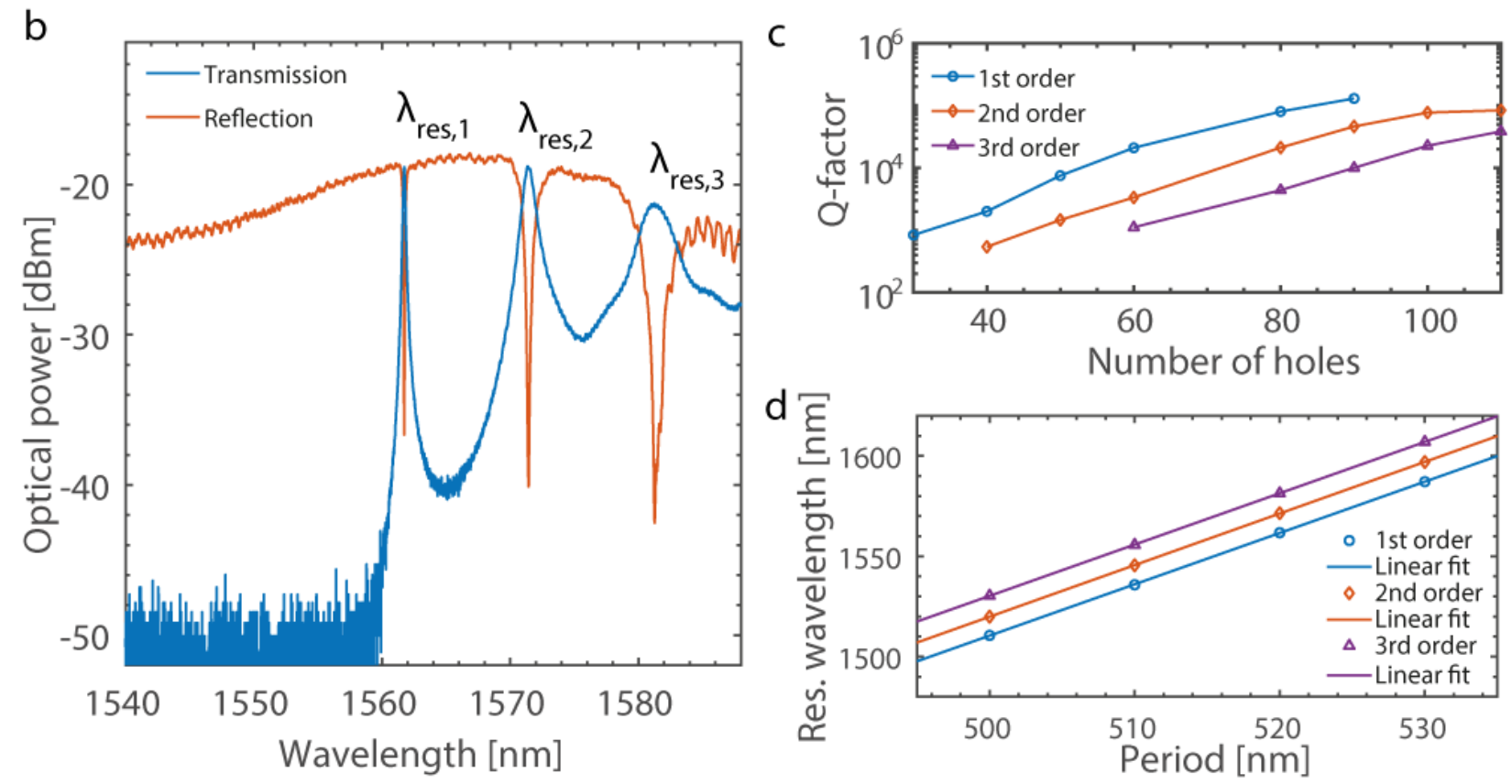

Figure 2. (a) Top view of the simulated electric field components within a bare photonic crystal cavity without GST. The first order resonance mode shows a Gaussian shaped field profile along the cavity which is a requirement for a high-Q cavity. Odd modes have a maximum in the center of the cavity, while even modes have a minimum. (b) Resonance modes show peaks and dips in the transmission and reflection spectrum of a fabricated device. (c) Fabricated devices show an exponential increase of the Q-factor in dependence of the number of holes per side and that the resonance wavelength can be tuned linearly by changing the period of the cavity (d). 

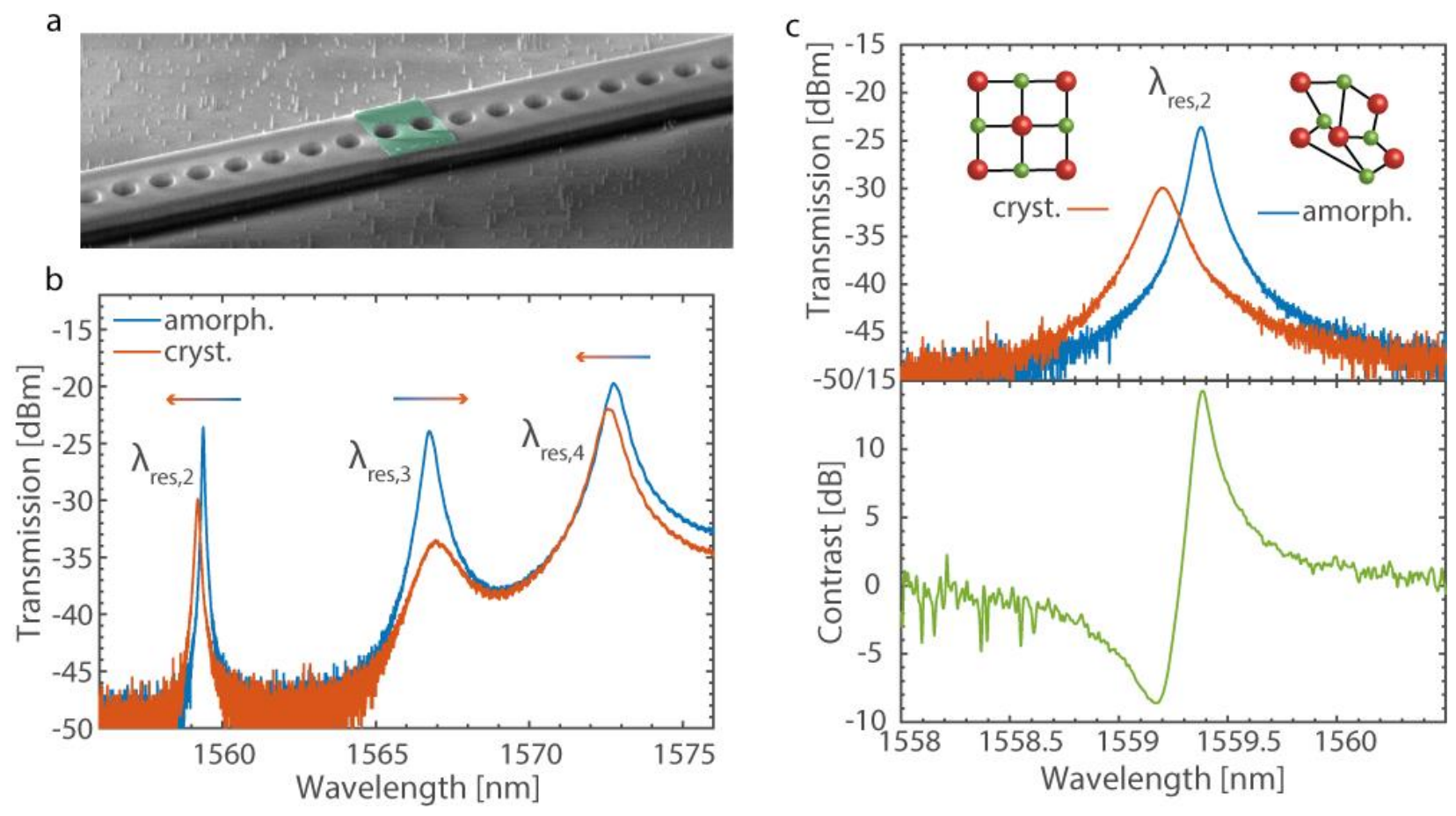

Figure 3. (a) SEM micrograph of a fabricated device with a $0.92 \mu \mathrm{m}$ long GST cell colored in green. The period, i.e. the distance between adjacent holes is $520 \mathrm{~nm}$. (b) Comparison of the transmission of resonance modes $\left(2^{\text {nd }}-4^{\text {th }}\right.$ order $)$ for a fully crystalline and amorphous cell. (c) Magnification of the transmission of the second order resonance mode and the wavelength depended contrast in transmission between an amorphous and crystalline GST cell exhibiting contrast of up to $14 \mathrm{~dB}$. 
a

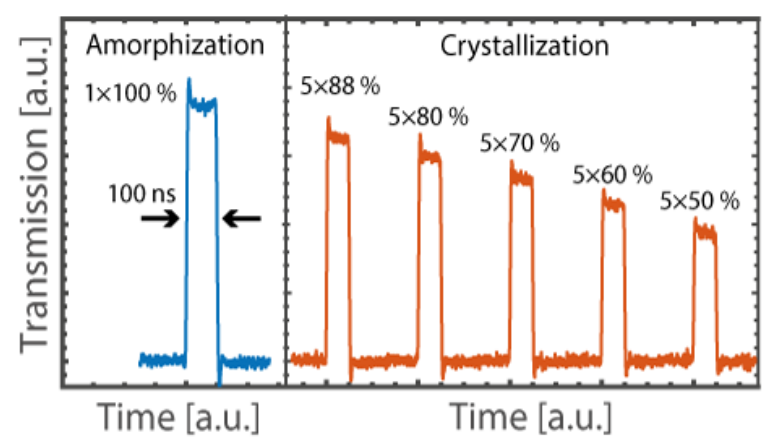

c b

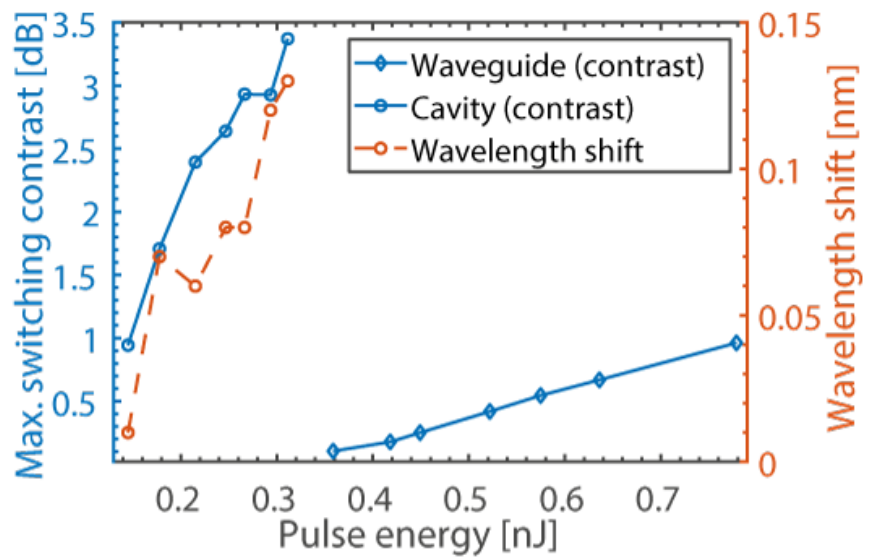

Figure 4. (a) Switching scheme of optical pulses (100 ns width) in order to amorphize and crystallize the GST cell. The switching pulses were sent on resonance at a wavelength of $1555 \mathrm{~nm}$, while readout is performed at a wavelength of $1542 \mathrm{~nm}$. (b) Maximal switching contrast and wavelength shift in dependence of the pulse energy used to amorphize the GST cell. The contrast for a GST cell embedded in a cavity clearly exceeds the values for a cell on solely a waveguide. (c) The switching contrast is constant for more than 2200 switches between the upper and lower transmission states. 